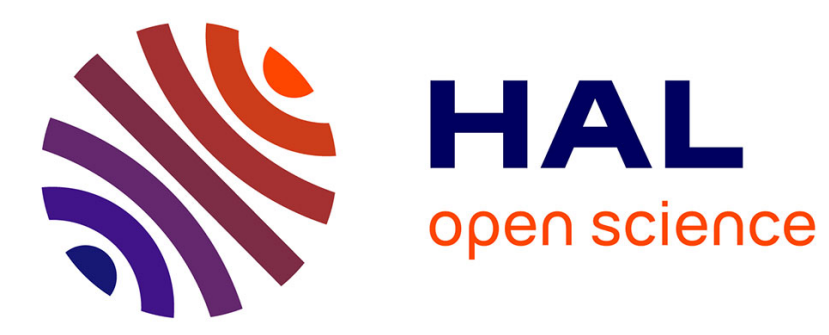

\title{
Barrages filtrants et correction torrentielle par ségrégation des matériaux charriés
}

\author{
Lucien Clauzel, André Poncet
}

\section{To cite this version:}

Lucien Clauzel, André Poncet. Barrages filtrants et correction torrentielle par ségrégation des matériaux charriés. Revue forestière française, 1963, 4, pp.280-292. 10.4267/2042/24540 . hal-03389993

\section{HAL Id: hal-03389993 \\ https://hal.science/hal-03389993}

Submitted on 21 Oct 2021

HAL is a multi-disciplinary open access archive for the deposit and dissemination of scientific research documents, whether they are published or not. The documents may come from teaching and research institutions in France or abroad, or from public or private research centers.
L'archive ouverte pluridisciplinaire HAL, est destinée au dépôt et à la diffusion de documents scientifiques de niveau recherche, publiés ou non, émanant des établissements d'enseignement et de recherche français ou étrangers, des laboratoires publics ou privés. 


\title{
BARRAGES FILTRANTS \\ ET CORRECTION TORRENTIELLE PAR SEGREGATION DES MATERIAUX CHARRIES
}

\author{
PAR \\ L. CLAUZEL \\ Ingénieur des Travaux des Eaux et Forêts \\ et \\ A. PONCET \\ Ingénieur en Chef des Eaux et Forêts
}

\section{INTRODUCTION}

Les problèmes connexes de la filtration des matériaux transportés par les torrents et d'une correction torrentielle par rétention sélective de ces matériaux préoccupent depuis longtemps en France et à l'étranger certains chercheurs.

Diverses solutions techniques ont été expérimentées avec des fortunes variées. La Revue Forestière en a rendu compte en son temps à ses lecteurs sous la plume autorisée du regretté conservateur P. Reneuve (1), mais de nouveaux essais ont été tentés.

Les objectifs pòursuivis par les chercheurs ou expérimentateurs sont d'ailleurs de deux ordres bien différents:

$1^{\circ}$ Souci d'une bonne résistance statique de barrages de correction torrentielle allégés et réalisés économiquement.

Il s'agit avant tout de soustraire les constructions transversales de rétention sédimentaire que sont les barrages aussi bien aux effets dynamiques ou de choc des laves pendant leur construction, d'où la conception d'ouvrages à élévation progressive au fur et à 
mesure de l'atterrissement, qu'aux effets temporaires ou permanents lès pressions hydrostatiques. Celles-ci engendrent, en effet, des poussées en moyenne deux fois plus fortes qu'une simple poussée cles terres sur le parement amont d'un ouvrage convenablement drainé. Emprisonnées derrière un ouvrage imperméable, elles exercent en outre des effets sournois sur les fondations. D'où l'idée de barrages à claire voie, à effet de grille ou de peigne, voire même cle simple nasse à graviers, dont la retenue se comble progressivement par filtration des matériaux charriés sans que l'ouvrage ait jamais à subir directement les effets d'une mise en pression hydrostatique. L'atterrissement grossier initialement fixé ne tarde pas à se colmater de limons fins, mais en conservant, grâce à son ossature graveleuse une cohésion et un coefficient de friction internes satisfaisants même en état de saturation hydraulique.

$2^{\circ}$ Principe d'une correction torrentielle réalisable, à titre temporaire ou définitif, par simple ségrégation ou rétention sélective des matériaux de l'ablation dans les lits torrentiels.

De telles recherches peuvent s'inspirer de l'optique traditionnelle des correcteurs de torrents disciples de Demontzey et Thiery qui consistait, selon l'heureuse formule de M. Widmann, à obtenir que les torrents débitent en clétail et sans dommages les matériaux de l'érosion dans leurs émissaires fluviaux chargés de les évacuer.

Or, ces émissaires, s'ils se chargent aisément de l'évacuation des limons, sables et même petits graviers, sont en général dangereusement engorgés par des matériaux plus grossiers. Ce sont donc ces (lerniers yu'il convient de retenir seuls dans les bassins torrentiels. Certains auteurs (M. Giandottr) n'ont-ils pas au surplus fait ressortir l'utilité d'une telle sélection pour laisser aux rivières une charge suffisante en limons nécessaires aux colmatages, ceux-ci permettant de constituer dans le lit majeur des rivières, dans les leltas fluviaux et même le long de certains rivages marins, de riches terres de culture?

Mais cette optique réaliste se heurte aux nouvelles conceptions d'une correction torrentielle intégrale envisagée sous l'angle de la lutte contre la sédimentation des grands réservoirs (*). Et les tenants de ces nouvelles conceptions ne se font pas faute de faire remarquer que les barrages dits filtrants ne filtrent pas longtemps, car les matériaux grossiers bloqués en premier lieu derrière la grille ou le peigne sont rapidement colmatés par des éléments de plus en plus fins jusqu'à constituer un écran imperméable.

(*) Correction torrentielle intégrale, entreprise sans doute chimérique aux yeux des forestiers affrontés aux puissants bassins d'érosion concentrée des Alpes, mais objectif idéal de recherches techniques digne de considération, au siècle de l'énergie atomique et des voyages interplanétaires. 
Le tri des matériaux transportés, retenant les gros et laissant écouler les fins avec le flot, doit, pour être efficace, être assuré de manière durable. Et les conditions mêmes des transports solides dans les torrents, révélées par l'étude des sédiments déposés, d'une granulométrie en général fort dispersée, laissent entendre qu'il serait fort hasardeux de se reposer sur une sélection de gros matériaux naturellement déposés ou artificiellement bloqués pour laisser filtrer les fins avec le courant à travers leurs interstices.

Certes S. Rosic (2) a cité des cas d'extinction spontanée de torrents par ensevelissement de leur lit sous de gros débris et substitution d'un inferoflux entièrement filtré et inoffensif aux écoulements superficiels érosifs. Mais les petits bassins torrentiels dans lesquels il a reconnu et décrit le phénomène, ont un substratum rocheux assez filtrant, dont la désagrégation semble produire plus d'éléments grossiers que d'éléments fins. Nous serions curieux de voir fareil phénomène se produire sur un substratum marneux ou argileux, voire schisteux ou de dépôts fluvio-glaciaires, avec ablation prédominante sous la forme d'éléments fins.

M. l'Ingénieur Clauzel, qui a déjà acquis une assez longue expérience de correction torrentielle sur de semblables terrains dans le département des Hautes-Alpes, s'est attaché à résoudre le problème de la ségrégation des matériaux grossiers et fins d'une manière efficace et durable par des artifices techniques encore inédits.

Sa première réalisation expérimentale dans un jeune torrent mixte de l'Embrunais (Clappes de Flysh gréseux et affouillement de dépôts glaciaires) semble bien donner depuis bientôt trois ans les résultats escomptés par son auteur, sans frais d'entretien appréciables.

Aussi lui ai-je proposé d'en informer lui-même des lecteurs de la Revute, en accord avec son chef de service M. l'Ingénieur RogIE à Gap, qui a assumé avec bienveillance sa part de responsabilités dans l'entreprise.

A. Poncet.

\section{II. - Compte Rendu D'Un Essai}

Nous avons réalisé en 1959, dans une ravine de la Commune de Saint-Clément, proche d'Embrun, un dispositif nouveau de correction torrentielle.

Les résultats de cet essai sont concluants, quoique perfectibles. Nous jugeons donc opportun de faire connaitre ce dispositif ; nous serions heureux qu'il provoque de la part des forestiers, en particulier de ceux qui s'occupent des questions torrentielles, des remarques ou des critiques. 
Critiques qui auraient pour résultats, surtout si elles sont suivies d'essais, d'améliorer et de rendre plus efficace encore ce moyen de lutte contre les torrents.

\section{Rappel de quelques principes théoriques}

I1 est peut-être bon de rappeler quelques principes théoriques pour mieux comprendre le raisonnement qui nous a amené à la conception de cet ouvrage.

Dans tout torrent, qu'il soit ou non à laves, l'eau joue un triple rôle: celui de moteur, de porteur, de lubrifiant.

L'action motrice de l'eau est évidente; elle se conjugue avec la pente longitudinale du lit du torrent.

Le rôle porteur de l'eau est tout aussi certain: une pierre immergée est plus facile à déplacer qu'une fois hors de l'eau. Les laves torrentielles ont une densité nettement plus grande que l'eau pure, voisine du double. Un torrent à laves aura donc un pouvoir de transport plus grand qu'un torrent à eau.

Quant au pouvoir lubrifiant de l'eau, il est amplifié par la présence de limons; des galets couverts de limons frais sont beaucoup plus glissants que simplement mouillés.

Si donc, dans un torrent, par un dispositif convenable, nous arrivons à prélever dans la lave ou dans la masse des matériaux charriés, une quantité notable d'eau et de limons, nous augmenterons la viscosité de la masse transportée. Plus secs, les éléments solides seront moins mobiles.

Cette “ ponction » réduira donc la vitesse d'écoulement du flot torrentiel; ce freinage doit permettre aux matériaux les plus volumineux et les plus lourds de s'arrêter, car le plus souvent, ces gros matériaux sont à la limite des possibilités de transport de la lave ou de la crue. Une réduction, même faible, de la force motrice de la crue, doit provoquer leur dépôt.

\section{Description du dispositif}

Obligeons le torrent à couler dans un chenal maçonné, d'une largeur sensiblement égale à celle de son lit 'moyen. Ce chenal sera limité sur les deux berges par des murs verticaux disposés selon un double entonnoir (voir plan d'ensemble).

Dans la partie inférieure de ce chenal, celle qui va s'évasant vers l'aval, disposons une grille métallique posée à plat ou avec une légère pente dans le même sens que celle du torrent (6 à $15 \%$ ) coupe E.F -

Sous cette grille filtrante, creusons un chenal dans lequel s'écouleront tous les produits d'essorage de la lave. Ce chenal aboutira à l'extrémité aval de la zone de dépôt que l'on s'est fixée. 
Les matériaux solides, d'un calibre supérieur à l'espacement des barreaux, ne pourront filtrer sours la grille et seront évacués vers l'aval, dans la zone de dépôt, grâce à l'énergie restante de la lave.

Les eaux, les limons et les matériaux d'un calibre inférieur à l'espacement des barreaux pourront filtrer sous la grille. L'essorage sera d'autant plus énergique que:

a) la surface de la grille sera plus grande,

b) la pente de la grille, plus faible,

c) l'espacement des barreaux, plus accusé.

Nous avons donc trois variables sur lesquelles nous pouvons jouer, soit isolément, soit simultanément, pour obtenir l'essorage que nous jugeons nécessaire.

Pour éviter tout danger d'obstruction de la grille par coincement, entre les barreaux, de cailloux d'un calibre proche de leur espacement, on donne aux éléments de cette grille une double divergence :

a) de l'amont vers l'aval, comme les nervures d'un éventail,

b) de haut en bas, les barreaux ont une section trapézoïdale la grande base du trapèze étant à la partie supérieure. -

Nous remarquons que, dans ce dispositif, la zone de dépôt se trouve à l'aval de l'ouvrage entre la grille et le point d'aboutissement du chenal d'évacuation. Notons que les conditions locales peuvent, parfois, permettre la dérivation dans un autre torrent. Cette solution est facilitée par le fait que la galerie de dérivation, ayant une section réduite puisqu'elle ne recevra qu'une partie des matériaux transportés et ceux les plus mobiles et les moins volumineux, sera d'un coût moins élevé que si elle devait recevoir la totalité de la crue.

Un ouvrage conçu sur ce principe a été réalisé à titre d'essai dans une ravine de la Commune te Saint-Clément, proche d'Embrun.

\section{La ravine de Combe-Crose ou de Pinfol}

Cette ravine, dans sa partie supérieure, est un véritable dérochoir. Pour une distance horizontale de 1770 mètres environ, la dénivelée est de 850 mètres, soit une pente longitudinale moyenne de $48 \%$. Les ouvrages classiques ne peuvent être efficaces que s'ils sont extrêmement nombreux, mais le coût global est prohibitif. Localement, les très grandes difficultés d'accès rendent la rentabilité des travaux encore plus discutable; il n'y a pas de zones d'érosion mais plutôt une érosion linéaire des berges du ravin, avivées à chaque passage d'une crue.

Dans sa zone moyenne, le ravin a un profil en long par ressauts. A des décrochements très raides succèdent des zones à faible 


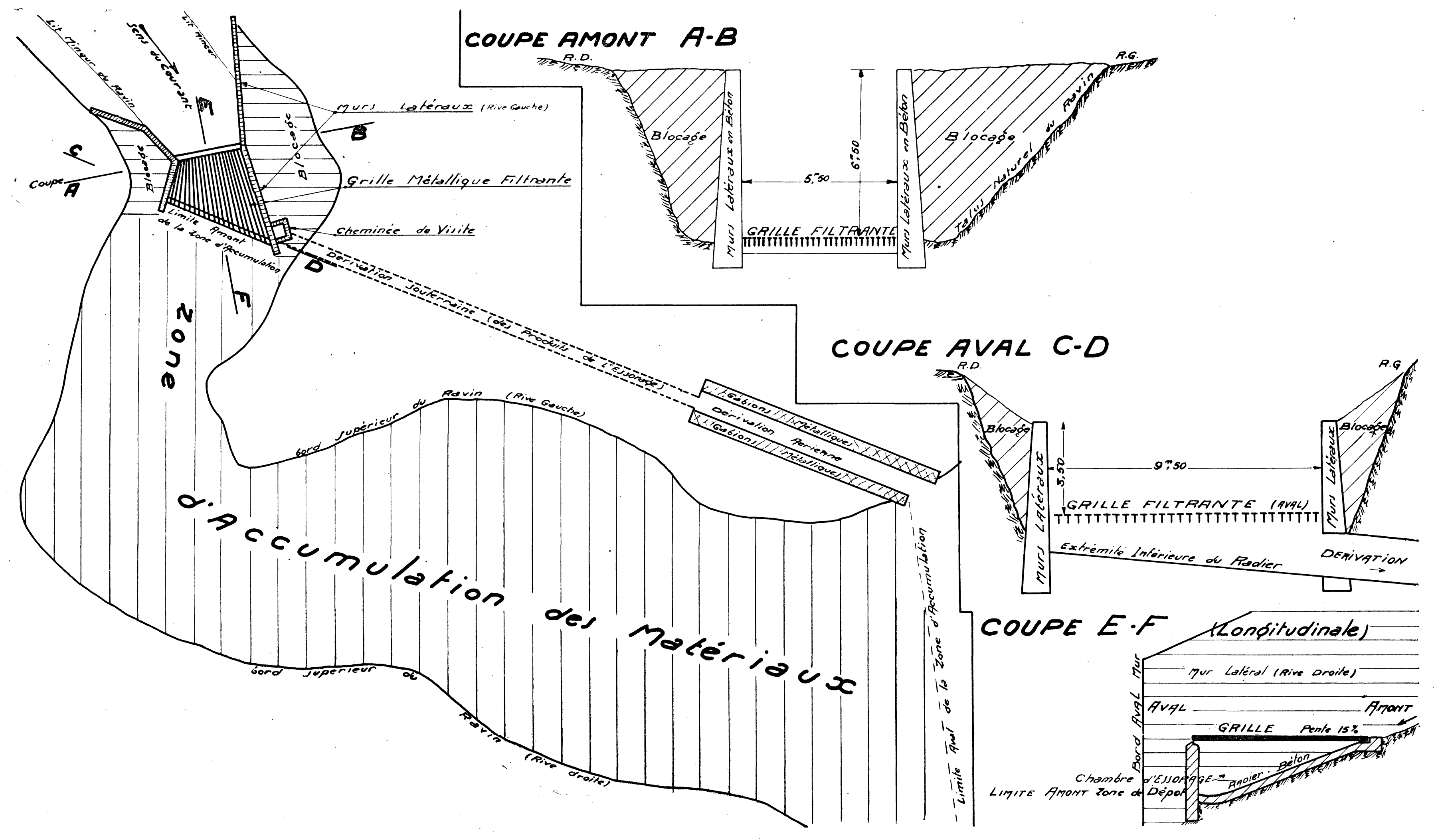




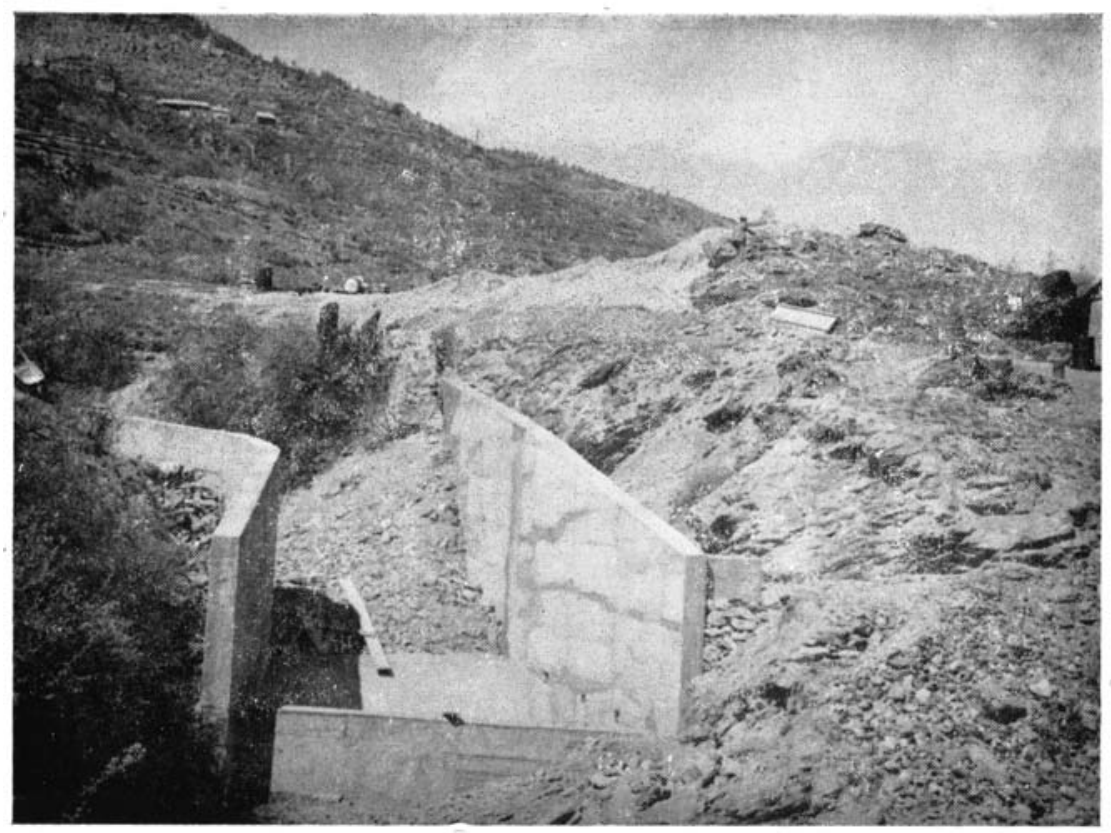

FIG. 1.

Construction du barrage-filtre de Combe-Crose, vue d'aval. Les bétons sont terminés. On distingue les murs latéraux du goulot, le mur aval supportant l'extrémité de la grille, le radier de glissement.

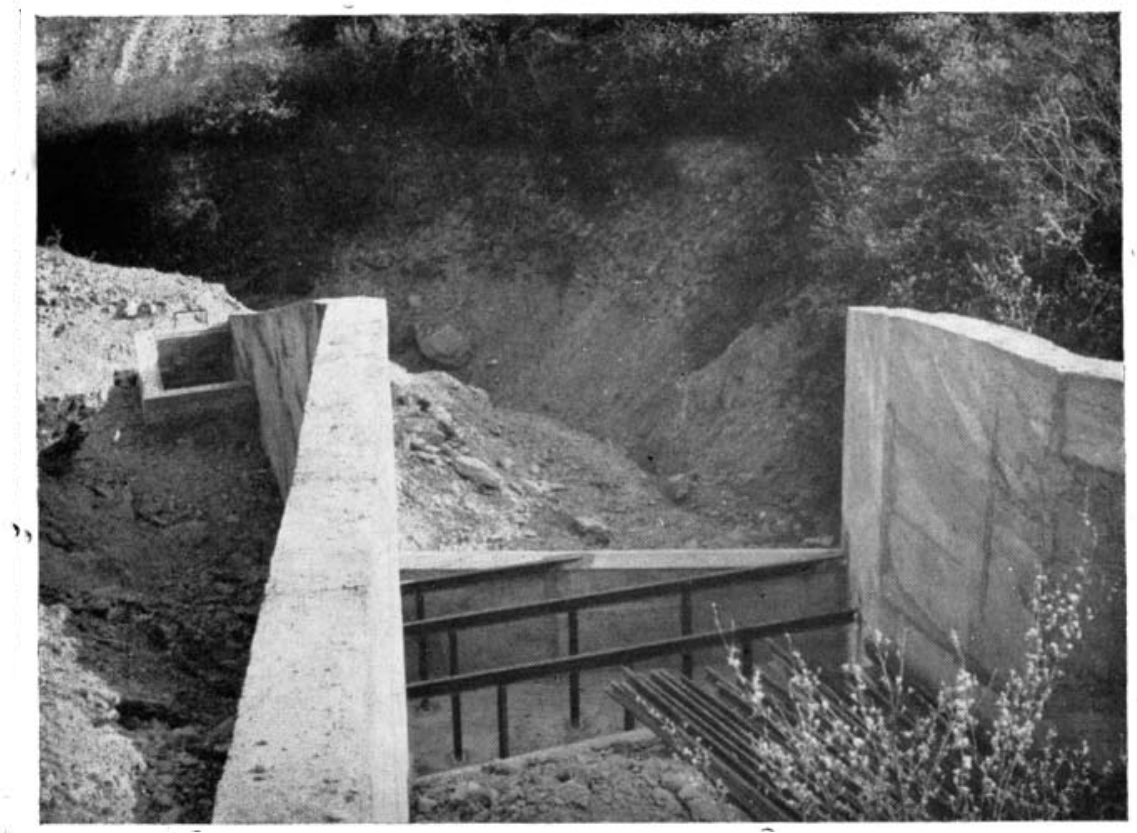

FIG. 2.

Construction du barrage filtre de Combe Crose, vue d'amont. Fosse de décantation. Pose des supports intermédiaires de la grille. A l'extrême gauche, le puits pour la visite de la grille. 


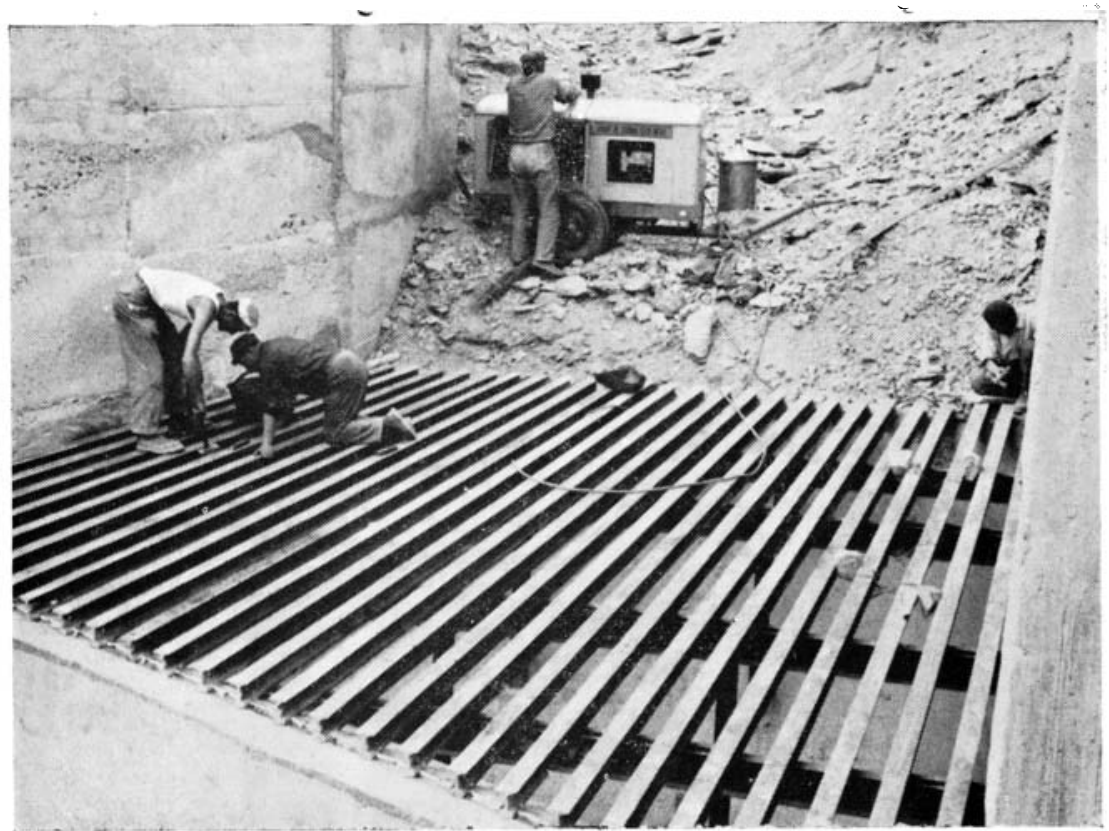

FIG. 3.

Pose de la grille; les ouvriers soudent les taquets latéraux maintenant à l'écartement prévu les barreaux de la grille; soudure à l'arc par poste électrogène mobile.

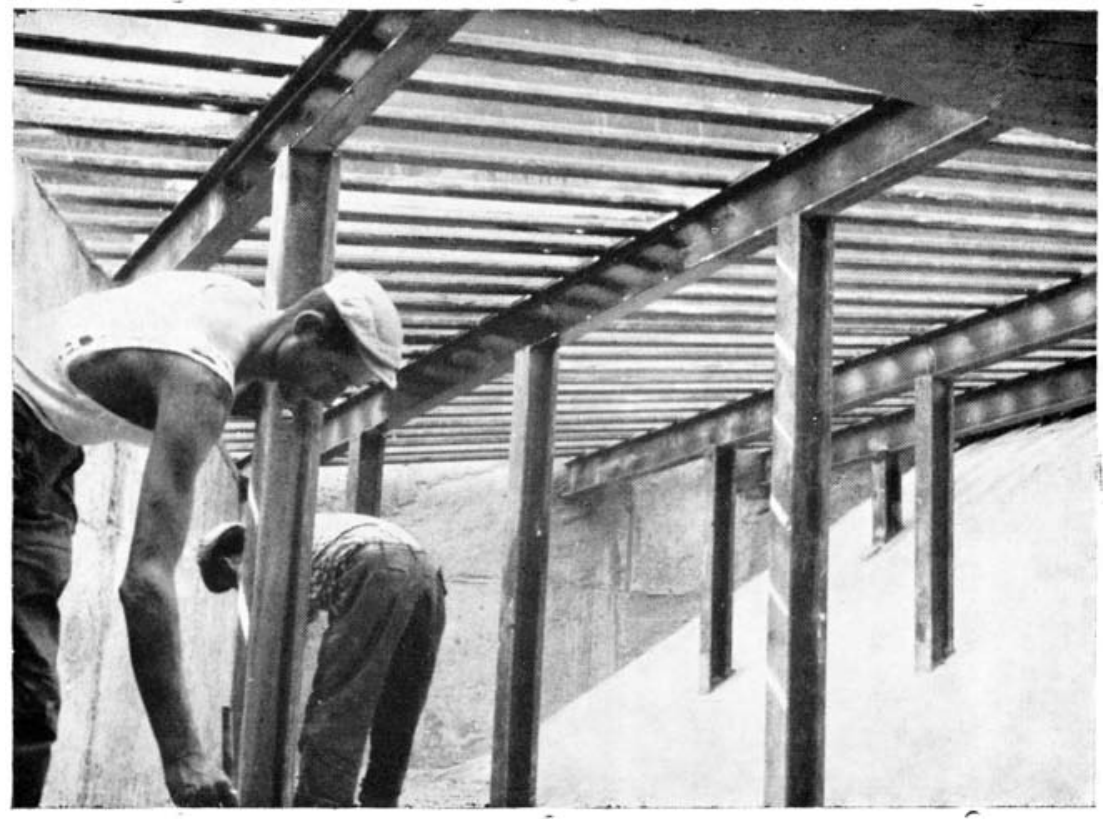

FIG. 4.

La grille vue de la chambre de décantation; à droite, le radier de glissement.

à gauche, le mur aval.

(Clichés Clauzel.) 
pente où le ravin est peu encaissé, presque au niveau des cultures qui ont colonisé ces replats. C'est au dernier de ces replats que nous avons édifié le barrage expérimental. De ce point jusqu'à la Durance, le ravin reprend une pente très forte.

Sur ces replats, nous avons constaté la présence de nombreux blocs laissés far les dernières crues. Ces blocs sont en cours de migration vers l'aval; ce sont eux qui, par leurs dimensions exceptionnelles (15 $\mathrm{m}^{3}$ parfois) obstruent les ponts et provoquent des débordements sur les voies de communication et sur les cultures.

La voie ferrée Paris-Briançon a été obstruée plusieurs fois, des déraillements se sont produits. Les Services Techniques de la S.N.C.F. envisageaient une modification du tracé sur plusieurs kilomètres, mais les conditions topographiques locales rendent cette dérivation très onéreuse, ce qui en a fait remettre l'exécution.

Nous avons donc, dans cette ravine, des conditions suffisamment dures pour être concluantes, mais à une échelle assez réduite pour que le coût de cet essai ne soit pas trop élevé. Un double coude brusque du torrent, au point choisi, s'il a compliqué la réalisation de la grille et en réduit peut-être l'efficacité, a pour avantage d'écourter la longueur du chenal de dérivation tout en permettant une zone de dépôt importante (voir plan d'ensemble).

\section{Réalisations - Détails techniques}

La grille constitue l'une des parties principales de ce genre d'ouvrage. A Combe-Crose, cette grille est constituée par des rails usagés de la S.N.C.F. - type P.L.M. (photo $\mathrm{n}^{\circ} 3$ ); ces rails ne sont pas posés dans le sens habituel, mais à l'inverse; le boudin du rail étant à la partie inférieure, le patin à la partie supérieure où il constitue la surface de glissement.

Cette disposition, peu orthodoxe, permet d'obtenir la double divergence des barreaux de la grille nécessaire pour éviter tout coincement des pierres entre les barreaux. La divergence horizontale est obtenue en donnant aux rails un espacement de $10 \mathrm{~cm}$ à l'amont et de $15 \mathrm{~cm}$ environ à l'aval. La divergence verticale résulte de la différence de largeur entre le boudin et le patin du rail.

Les rails sont soudés électriquement à l'amont sur une cornière noyée dans le béton. Mais sur les supports intermédiaires et aval (photo $\mathrm{n}^{\circ} 2$ ), ces rails reposent librement sur les appuis. Il n'y a ni boulons ni écrous ni entretoises qui pourraient provoquer l'arrêt des cailloux entre les rails.

Les espacements prévus sont obtenus par des cales en fer carré d'une hauteur égale à celle du boudin du rail - cales qui sont soudées sur les supports.

Sous la grille, un radier de glissement (photo $\mathrm{n}^{\circ} 4$ ) en béton facilite l'évacuation des produits de l'essorage. Quant au chenal 
d'évacuation, il est tracé dans le prolongement du mur inférieur du radier, ceci pour réduire au maximum les remous, les turbulences qui pourraient provoquer un arrêt ou un dépôt des produits de l'essorage.

A Combe-Crose, la réalisation de ce chenal a été difficile. La roche s'est révélée, au cours des travaux, très disloquée; il a fallu

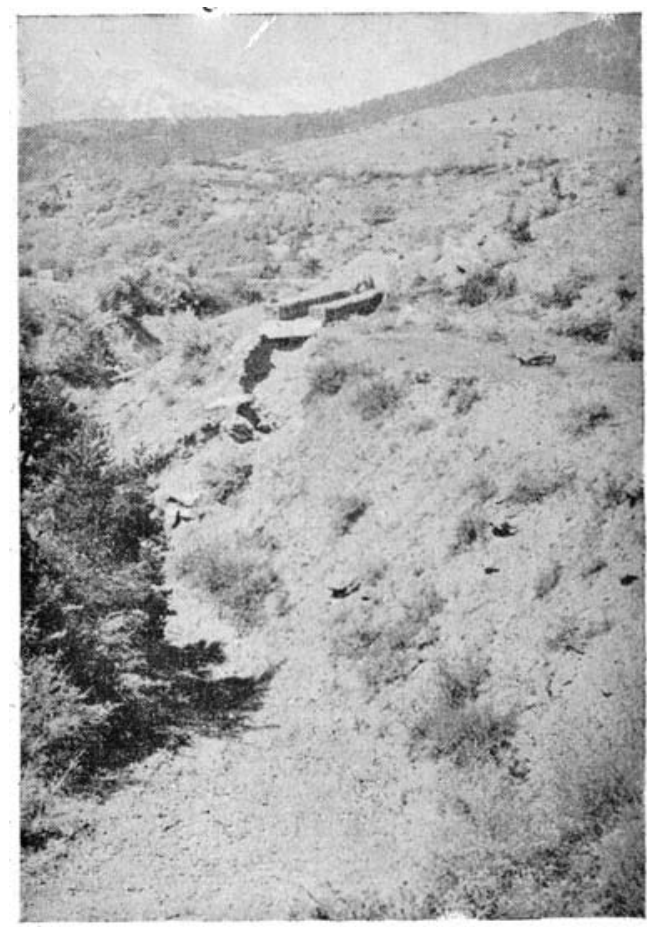

Fig. 5.

Ravin de Combe-Crose. Le point d'aboutissement du chenal de dérivation, à quelque $50 \mathrm{~m}$ en aval du barrage-filtre. Les produits de l'essorage sont très abondants, d'où un affouillement très marqué. Depuis la date de ce cliché, 22 avril 1961, il a fallu construire de nouveaux gabions pour protéger du déchaussement la murette terminale. Ces gabions sont à leur tour déchaussés, ce qui prouve l'efficacité de l'essorage et le bon fonctionnement de la grille. La chute de restitution au torrent du flux essoré et érosif nécessiterait un aménagement moins sommaire, par exemple un bec verseur surplombant, en béton armé.

(Cliché Clauzel.)

creuser le chenal à ciel ouvert et substituer à la galerie initialement envisagée une conduite faite avec des buses de $1 \mathrm{~m}$ de diamètre. Cette conduite a une section presque moitié de celle prévue, et, quoique cette section circulaire ait, au point de vue hydraulique, un rendement supérieur à une section carrée ou rectangulaire, elle 
semble, à Saint-Clément, un peu insuffisante. Au passage des laves, ces buses débitent à pleine section et avec pression. Des traces de boues, jusqu'à une assez grande hauteur dans la cheminée de visite, prouvent que la grille essore plus de matériaux que les buses. ne peuvent en évacuer. L'usure de la grille doit s'en trouver réduite, mais l'essorage est moins énergique.

Les murs latéraux constituant les parois verticales du double entonnoir sont en béton. Un blocage en tout venant de Durance comble le vide entre ces murs et les anciennes berges du ravin.

Une cheminée de visite permet d'accéder facilement dans la chambre d'essorage, sous la grille. Il est, comme cela, facile et possible de vérifier l'état de la grille, du radier de glissement, du départ du chenal d'évacuation tout en facilitant les réparations qui peuvent être nécessaires.

Remarque. - Rien ne s'oppose à ce que des grilles comparables à la grille horizontale soient établies le long des parois verticales du chenal bétonné; ces grilles verticales essoreraient latéralement la lave. Mais il semble inutile de vouloir essorer la lave au maximum - sauf dans le cas où le dispositif est édifié en tête d'un à pic, d'un dérochoir.

Un essorage partiel freine seulement la lave. Il suffit que ce freinage soit assez énergique pour provoquer l'arrêt des matériaux dans la zone de dépôt fixée.

\section{Prix de revient}

Il est difficile de donner, pour ce type d'ouvrage, un prix de revient moyen. Un des postes important de dépenses est le chenal d'évacuation. Le coût de ce chenal varie considérablement selon les conditions locales: longueur de la dérivation, section, conditions dans lesquelles elle sera faite: en galerie, à ciel ouvert, etc...

A Combe-Crose, la galerie de fuite a une longueur de 30 mètres en galerie busée et de 15 mètres à ciel ouvert. Quant à l'ouvrage proprement dit, il est en général d'un prix de revient comparab!e, sinon inférieur à un ouvrage classique. En effet, la grille est la partie la plus onéreuse, mais l'emploi de rails de rebut de la S.N.C.F. permet de la réaliser à des conditions de prix très acceptables.

Quant aux murs latéraux, ils peuvent être construits très simplement. La poussée du torrent étant presque entièrement équilibrée par les remblais que l'on met entre les murs et les berges même du torrent.

A Combe-Crose, un ouvrage classique, en béton armé, construit par la même entreprise, et faisant partie du même marché, est revenu plus cher que l'ouvrage expérimental (56000,00 NF contre $46000,00 \mathrm{NF}$ au (" barrage grille )). Sa capacité de rétention est pourtant de plusieurs fois inférieure à celle du barrage grille. 
Remarquons de plus, à l'avantage du barrage expérimental, qu'il est possible, à Combe-Crose, de prolonger le chenal d'évacuation de plus du double de sa longueur actuelle. Ici, cette prolongation peut se faire à ciel ouvert; le coût en serait faible; le volume de la zone de dépôt serait quintuplé.

\section{Conclusions}

L’originalité du dispositif réside donc dans le fait qu'il retient les matériaux à son AVAL et non à l'amont comme dans tous les dispositifs habituels. Cette particularité présente les avantages suivants :

$1^{\circ}$ il doit être possible d'utiliser les cônes de déjection comme zone de dépôt, des matériaux les plus gênants;

$2^{\circ}$ l'efficacité du barrage filtrant est d'autant plus grande que la pente du lit du torrent est plus forte à l'aval du barrage. Les dangers d'obstruction de la grille sont réduits et le volume de la zone de dépôt est plus important;

$3^{\circ}$ la dérivation d'un torrent doit pouvoir se réaliser, dans de bien meilleures conditions. La galerie ou le chenal de dérivation auront une section nettement plus faible car il n'y sera dérivé que les produits d'essorage, ceux les plus mobiles et les moins volumineux. L'usure même de cette dérivation sera plus faible, les produits les plus abrasifs étant dirigés vers la zone de dépôt à l'aval de la grille.

Ce "système qui fonctionne à l'inverse des barrages habituels trouve ses conditions optima là où justement les méthodes classiques sont économiquement inapplicables. Il n'est donc nullement destiné à les supplanter ou à les concurrencer, mais il complète plutôt nos moyens de lutte en apportant une solution là où les conditions. 10cales rendent les procédés classiques inefficaces ou trop onéreux.

L. Clauzel.

\section{III. - Commentaires}

Les conclusions de l'inventeur du dispositif expérimenté à Combe-Crose appellent quelques remarques.

Il est tout d'abord assez aisé d'interpréter l'action d'un tel dispositif en se référant à la théorie et aux formules des écoulements torrentiels développées par THIERY et BERNARD, après avoir remarqué qu'un tel ouvrage, à l'inverse des ouvrages classiques, entraîne à son aval un important relèvement de la pente de compensation, qui peut atteindre passagèrement, après une petite crue, la valeur d'une pente de talus naturel d'éboulis. Souvenons-nous que la formule d'une pente de compensation instantanée I fait intervenir à son dénominateur le carré d'un coefficient de torren- 
tialité $\mathrm{K}$, introduit dans la formule de CHezy. Ce coefficient est d'autant plus inférieur à l'unité que le courant est plus chargé en matériaux solides. Un essorage ou une décantation fartielle à travers une grille laisse s'écouler en profondeur un courant très peu chargé ( $K$ voisin de 1 ) qui pourra se contenter d'un chenal de dérivation de faible pente. Par dessus la grille, au contraire, les matériaux grossiers ne seront déblayés que par les excédents liquides des pointes de crue. Il en résultera des écoulements excessivement chargés ( $K$ très faible) susceptibles de se déposer sur une pente d'autant plus forte que la grille aura absorbé plus complètement le débit liquide et de matériaux fins lubrifiants. Les qualités hydrauliques des profils de l'ouvrage, la pente et les dimensions de la grille, l'écartement de ses barreaux, le gabarit de la dérivation souterraine d'évacuation des liquides et produits fins doivent donc permettre de régler autour d'une valeur moyenne convenable les pentes de compensation susceptibles de s'établir à l'aval de l'ouvrage. Ces considérations nous aident à comprendre la première et plus surprenante conclusion de l'auteur: l'utilisation possible, grâce au dispositif, des cônes de déjection comme zone de dépôt des matériaux les plus gênants.

Chacun sait qu'un cône de déjection au terme de sa formation a ses génératrices inclinées avec la pente de compensation moyenne minima de tout le parcours torrentiel. Un dispositif analogue à celui cle Combe-Crose placé sur le cône, même à son sommet, aurait donc toute chance d'être rapidement ennoyé par les premiers matériaux grossiers décantés et bloqués à l'aval, et serait donc rendu inopérant.

Il est vrai toutefois que rares sont les cones complètement formés. Beaucoup, heureusement, n'en sont encore qu'au $2^{\mathrm{e}}$ ou $3^{\mathrm{e}}$ stade de leur formation et comportent sur leurs flancs des pentes largement supérieures à la pente de compensation moyenne du cours inférieur du torrent. Il est donc possible d'en envisager le rechargement. Mais la variabilité des écoulements torrentiels est bien connue des praticiens. Aux crues chargées et remblayantes succèdent des crues claires et érodantes aui se nourrissent par reprise d'érosion dans la plage de dépôt elle-même quand celle-ci a été établie sur une pente un peu excessive. Et ces recharges torrentielles peuvent aller se déposer et faire des dégâts au loin, au delà des limites d'une plage de dépôt insuffisamment endiguée. Cet inconvénient entraîna la nécessité de travaux importants de diversion et de blocage des laves dans la plage de dépôt d'un important torrent à Tappes de l'Isère (Les Palles - Chantelouve - 3).

M. Clauzel semble envisager surtout son dispositif comme un système d'essorage ou de décantation des laves. Ces écoulements torrentiels avec transport en masse sont les plus dangereux par leurs effets cinétiques et par les profonds surcreusements qui en résultent. Or, un ouvrage filtrant de ségrégation peut n'être conçu et 
dimensionné que pour essorer partiellement et provoquer un simple freinage des laves, suffisant pour entraîner la rupture de leur cohésion et leur arrêt par dispersion plus ou moins lointaine.

Un tel ouvrage, fortement canalisant, implanté au débouché de la gorge et au sommet du cône aurait moins de chances d'être enseveli par remblaiement remontant en matériaux grossiers, et verrait sa grille périodiquement dégagée par les fins de crues peu chargées en matériaux*.

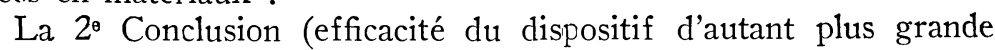
que la pente du torrent est plus forte à l'aval du barrage) traduit une évidence, qui prend toute sa valeur pour la correction d'un torrent encore jeune, échancrant peu profondément de très fortes pentes coupées de ressauts, tel la Combe-Crose.

La même considération rend le dispositif particulièrement intéressant pour la correction des torrents à clappes, généralement moins régulièrement et profondément encastrés dans les versants que les torrents à affouillement, correction qui ne procède guère que par rétention de matériaux grossiers. Or, les ( ombilics ) favorables à la rétention d'une masse importante de sédiments derrière un barrage classique sont rares. En rechargeant en matériaux grossiers filtrants les talwegs en forte pente à l'aval de dispositifs d'essorage. on peut non seulement bloquer dans la montagne une masse considérable de matériaux qui rechausseront les berges instables et en autoriseront le reverdissement, mais créer en certaines sections grossièrement remblayées les conditions favorables à un écoulement souterrain infiltré, solution idéale de la correction torrentielle chère à M. le Professeur Rosic.

Par ailleurs, le dispositif expérimenté à Combe-Crose, s'il semble bien assurer une séparation et un blocage efficace des gros matériaux de transport torrentiel, ne résoud pas, par lui-même, le problème de l'écoulement du liquuide résiduel de l'essorage, chargé seulement de matériaux fins. Or, cet écoulement non saturé est doué d'une puissance nette érosive fort appréciable. On ne peut donc le rejeter n'importe où dans un torrent très affouillable. Par contre, les torrents à clappes sont souvent développés dans des formations géologiques comportant une succession d'assises rocheuses dures et solides, alimentant les dérochoirs, et d'assises plus tendres sans lesquelles il n'y aurait pas de torrent. S'il convient d'organiser le blocage des matériaux grossiers rocheux dans les sections tendres et affouillables du lit torrentiel, il peut être possible également, dans

* Avec M. l'Ingénieur Rogie, nous croyons toutefois préférable d'implanter l'ouvrage plus haut que le sommet du cône, pour stopper les gros matériaux en premier lieu dans la partie inférieure de la gorge toujours plus déclive et permettant des fondations plus solides que le cône. Ainsi seraient réduits les risques d'obturation de la grille et de divagations torrentielles dangereuses pour une dérivation superficielle en terrain affouillable. 
de tels torrents, de rejeter les écoulements décantés exclusivement dans les sections rocheuses ou résistantes du lit... s'il s'en trouve à un niveau convenable.

Or, c'est pour les torrents à clappes, de pente moyenne génćralement très forte, que les méthodes classiques de la correction torrentielle par échelle de barrages pleins et massifs se révèlent bien souvent “ économiquement inapplicables ). En effet, l'atterrissement de chaque barrage doit rechausser les fondations du barrage supérieur. La correction intégrale du lit torrentiel entraine une multiplication considérable d'ouvrages d'une réalisation et d'un entretien très onéreux.

Au surplus, cette correction torrentielle, en raison des risques de léchaussement des barrages classiques par affouillement aval, procèdle obligatoirement de bas en haut à l'intérieur d'une section du cours torrentiel. Or, sur les fortes pentes qui caractérisent les ravins torrentiels, le reboisement est condi1it plus sûrement de haut en bas, les plantations inférieures bénéficiant de l'abri des plantations supérieures contre les effets de la reptation des neiges, des éboulis et de la solifluxion.

Marchant en sens inverse et à la rencontre l'une de l'autre, l'œuvre de la correction du lit torrentiel par les barrages classiques et celle clu reverdissement $\mathrm{du}$ bassin risquent de mettre fort longtemps pour se rencontrer et conjuguer leurs effets harmonieusement. Un dangereux hiatus subsistera entre elles si leurs programmes ont été mal coordonnés, faute de moyens ou faute d'une prévision du développement des processus d'érosion au cours d'une entreprise s'étendant le plus souvent sur plusieurs décennies. Ce hiatus se traduit ordinairement par la persistance de berges instables, non végétalisées et en voie d'érosion accélérée entre un lit corrigé par barrages mais trop encaissé et des peuplements forestiers dangereusement suspendus au sommet de berges vives. Cette lacune est très fréquente en France, même dans des torrents réputés corrigés.

Or, le barrage-grille expérimenté à Combe-Crose ne craint pas l'affouillement aval bien au contraire. Il peut donc être implanté d'emblée dans le haut des ravins ou au-dessus du point précis du lit torrentiel qu'il s'agit de remblayer pour rechausser des berges instables.

Mais le dispositif de Combe-Crose n'a pas encore révolutionné les méthodes classiques de correction torrentielle. Et son auteur se garde bien de toute généralisation imprudente. Seul un commentateur pouvait se permettre d'imaginer une exploitation de l'idée pour une correction intégrale menée de haut en bas de certains ravins torrentiels suffisamment riches en débris grossiers. Encore pense-t-il que ce dispositif ne saurait détrôner les barrages classiques à effet-amont simple et sûr, de rétention par soutènement, toujours préférables dans les sections évasées et à faible pente, en 
général très affouillables. Le dispositif expérimenté me parait donc à priori voué à plus de succès dans le haut d'un bassin torrentiel de quelque importance que dans le bas.

La $3^{3}$ conclusion de M. Clauzel est certainement, dans l'immédiat, la plus pratique. Toute dérivation torrentielle nécessite un barrage insubmersible résistant aux pressions hydrostatiques, donc massif et onéreux. Le tunnel ou le chenal de dérivation doit être calibré pour assurer les plus forts écoulements et les plus chargés en matériaux grossiers. Or, les écoulements les plus volumineux à débit égal, les plus érodants et les plus dangereux quant à un engorgement éventuel du tunnel ou du chenal sont les laves. Un barrage de dérivation submersible assurant la décantation des laves apparait donc comme le complément ingénieux d'une dérivation torrentielle économique, grâce à un gabarit réduit. Or, toutes les possibilités de correction par dérivation sont loin d'être exploitées dans les ravins de nos torrents alpins.

En définitive, l'ingéniosité de $M$. Clauzel me semble bien avoir doté l'arsenal des moyens techniques dont dispose le forestier correcteur de torrent d'un nouvel instrument permettant d'apporter immédiatement un frein aux phénomènes de torrentialité les plus gênants pour mener à bien une œuvre de correction torrentielle intégrale. Celle-ci restera sans doute toujours fondée sur les méthodes classiques, dont les ouvrages sont susceptibles de perfectionnement en fonction des progrès des matériaux et techniques modernes de construction.

A. Poncet.

\section{REFERENCES BIBLIOGRAPHIQUES}

1. P. Reneuve. - L'évolution de la technique de correction torrentielle. Revue Forestière Française, 1955, p. 689-693.

2. Sr. Rosic. - Quelques exemples de l'extinction spontanée des torrents. R.F.F., 1956 , p. $515-522$.

3. J. Cherrey. - Circulation des laves torrentielles sur les cônes de déjection. Voyage d'études dans les Alpes françaises, p. 230-235. Edition de l'Ecole Nationale des Eaux et Forêts, 1953. 